\title{
Dimensionamento ótimo de pórtico de aço com ligações semirrígidas utilizando elementos finitos híbridos
}

\author{
Sérgio da Silva Ferreira Júnior ${ }^{1 *}$ e Gines Arturo Santos Falcón ${ }^{2}$ \\ ${ }^{1}$ Laboratório de Engenharia Civil, Universidade Estadual do Norte Fluminense \\ Darcy Ribeiro, Av. Alberto Lamego, 2000, 2803-602, Campos dos Goytacazes - \\ RJ, ssferreira.jr@gmail.com \\ ${ }^{2}$ Laboratório de Engenharia Civil, Universidade Estadual do Norte Fluminense \\ Darcy Ribeiro, Av. Alberto Lamego, 2000, 2803-602, Campos dos Goytacazes - \\ RJ, gines@uenf.br
}

\section{Optimum steel frame design with semi-rigid connections using hybrid finite elements}

\begin{abstract}
Resumo
Apresenta-se uma metodologia computacional para dimensionamento ótimo de pórticos planos de aço utilizados em edifícios multi-andares. O estudo visa a definição de pórticos planos de aço eficientes e econômicos. Considera-se o comportamento não linear geométrico da estrutura e o comportamento semirrígido das ligações viga-coluna. As variáveis de projeto são as rigidezes das ligações e os perfis comerciais nacionais, variáveis continuas e discretas, respectivamente. As restrições de projeto são adotadas em conformidade com normativas locais. Foram desenvolvidos dois módulos computacionais integralmente no ambiente computacional MATLAB. Para análise estrutural, baseado no Método dos Elementos Finitos, foi implementado um Elemento Finito Híbrido que adiciona elementos tipo mola rotacional nas extremidades do elemento viga clássico. Exemplos são apresentados para ilustrar a metodologia proposta.
\end{abstract}

Palavras-chave: Pórtico de aço, Ligações semirrígidas, Otimização estrutural, Elemento Finito Híbrido.

\begin{abstract}
It presents a computational methodology for optimal sizing of plane steel frames used in multistorey buildings. The study aims at the definition of efficient and economical plane steel frames. It is considered the geometric non-linear behavior of the structure and the semi-rigid behavior of the beam-column connections. The design variables are the stiffness of the connections and commercial profiles available in the local market, continuous and discrete variables, respectively. The design constraints are adopted in accordance with local regulations. Two computational modules were developed integrally in the MATLAB computational environment. For structural analysis, based on Finite Element Method, was implemented a Hybrid Finite Element, which adds rotational spring elements at the ends of the classic beam element. Examples are presented to illustrate the proposed methodology.
\end{abstract}

Keywords: Steel frame, Semi-rigid connections, Structural optimization, Hybrid finite element.

* autor correspondente 


\section{Introdução}

No projeto clássico de pórticos de aço são adotados dois modelos hipotéticos de comportamento para as ligações segundo a sua rigidez rotacional: articulado ou rígido. Esses modelos são adotados visando a simplificação da análise estrutural, desconsiderando desta forma, o comportamento semirrígido que realmente ocorre nas ligações, Freitas (2010).

Neste contexto, as ligações viga-coluna desempenham um papel determinante na distribuição de esforços internos e consequentemente no comportamento mecânico de pórticos de aço, como atestam diversos estudos realizados nas últimas décadas.

Muitos estudos numéricos e ensaios foram realizados para verificação do comportamento mecânico de ligações semirrígidas. Dentre outros, Mesquita (2002) apresentou um banco de dados, denominado SERICON II, onde diversos ensaios realizados na época encontram-se devidamente catalogados. Simões (1996) e Díaz (2011) apresentaram estudos para minimização do custo de fabricação de pórticos de aço com ligações semirrígidas. Em geral, os trabalhos revisados utilizam diversas técnicas de análise estrutural e algoritmos de otimização. Observa-se o grande potencial do Método dos Elementos Finitos e dos Algoritmos Genéticos na solução de complexos problemas de otimização estrutural.

De acordo com Díaz (2011), nos pórticos de aço a ligação articulada permite a rotação relativa entre a viga e o pilar, portanto não há transmissão de momentos fletores entre esses elementos. Já as ligações rígidas, não permitem a rotação relativa entre esses elementos, causando então, transferência de momentos fletores entre vigas e colunas. No entanto, a característica real de uma ligação apresenta um comportamento intermediário entre os casos citados, denominada semirrígida, conforme mostra a Figura 1, onde $\phi$ a rotação entre a viga e a coluna. Para cada rigidez rotacional inicial $\left(S_{\text {ini,i }}\right)$ adotada, com o carregamento e o perfil inalterados, têm-se diferentes valores de rotação. 

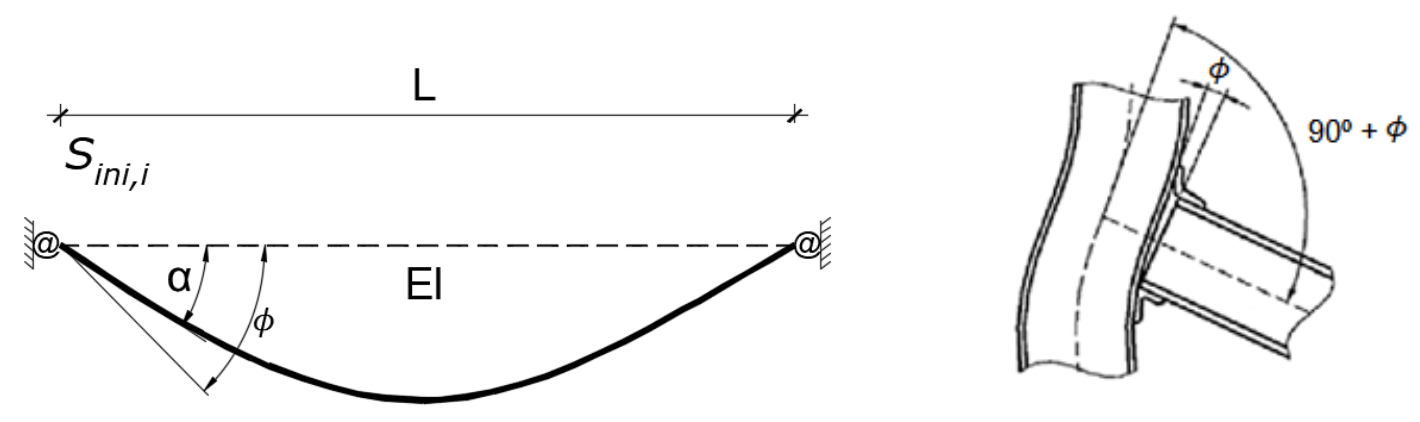

Figura 1 - Comportamento da ligação semirrígida.

A Figura 2 mostra o comportamento mecânico de uma ligação viga-coluna, em função do momento aplicado e da rotação relativa produzida por este momento, onde observase um trecho inicial linear elástico seguido de um trecho não linear. Nota-se que ligações articuladas representadas, por exemplo, pela ligação de cantoneira dupla, Figura 2 (a), apresenta elevadas rotações com pequenos momentos, diferente das ligações com significativa rigidez rotacional, representada, por exemplo, pela ligação de chapa de topo mostrada na Figura 2 (b), que se caracteriza por apresentar pequena deformação rotacional e ruptura frágil.

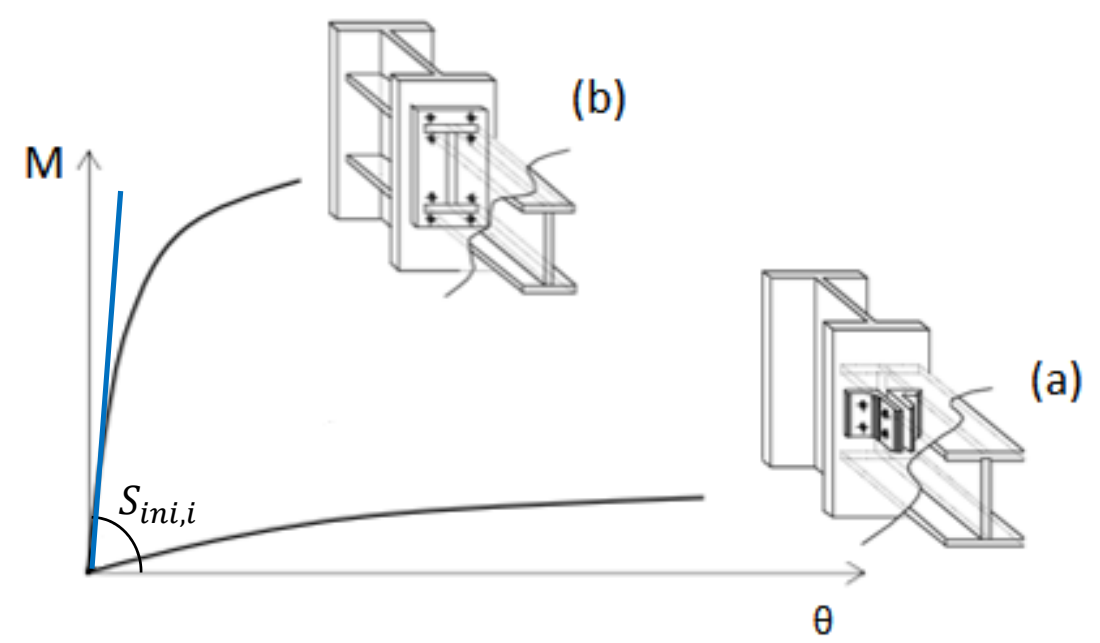

Figura 2 - Curva momento-rotação: (a) cantoneira dupla de alma; (b) placa de extremidade.

A característica da curva momento-rotação de uma ligação depende de suas propriedades de resistência à flexão, rigidez rotacional e capacidade de rotação (ductilidade). A rigidez rotacional inicial $\left(S_{\text {ini,i }}\right)$ define a relação linear entre o momento fletor solicitante e a rotação relativa na etapa inicial de deformação da ligação. Em função deste parâmetro, a ABNT NBR 8800:2008 define limites inferiores e superiores para classificação das ligações semirrígidas, Equação (11). 
Em virtude da influência significativa das ligações semirrígidas no comportamento dos pórticos de aço, principalmente em relação aos deslocamentos laterais, o presente estudo preocupa-se pela análise estrutural na sua posição deformada, isto é, análise não linear geométrica.

Assim, foram implementados dois módulos computacionais, um para análise do comportamento mecânico de pórticos planos de aço com ligações viga-coluna semirrígidas e outro para o dimensionamento ótimo, ambos os módulos implementados integralmente no ambiente computacional MATLAB.

O módulo computacional de análise estrutural foi implementado a partir do programa de domínio público CALFEM - "Computer Aided Learning of Finite Element Method" (Lund University, 2004). O CALFEM é um programa computacional utilizado em diversas universidades para o ensino de técnicas de elementos finitos. No entanto, o programa foi reformatado e aprimorado para seu uso automatizado no processo iterativo de otimização, utilizando interfaces baseada em arquivos de entrada de dados e saída de resultados. Desta forma, foram implementados uma versão para análise linear elástica e outra para análise não linear geométrica. O programa CALFEM foi adaptado para consideração das ligações semirrígidas no comportamento mecânico dos pórticos de aço através da inclusão de um elemento finito híbrido que aqui se propõe.

O modelo de otimização adotado objetiva reduzir o custo de fabricação de pórticos aço, considerando-se simultaneamente o custo dos perfis estruturais e o custo das ligações viga-coluna. As variáveis de projeto (VP) são os perfis estruturais (VP discretas) e o grau de rigidez rotacional das ligações (VP contínuas). Para o primeiro grupo de variáveis foi criado um banco de dados e suas interfaces computacionais, visando a definição automática da configuração estrutural no processo iterativo de otimização.

As restrições de projeto estão baseadas nas prescrições da ABNT NBR 8800:2008, com relação a deslocamentos e resistências mecânicas.

\section{Análise estrutural do pórtico de aço}

No módulo de análise estrutural desenvolvido, propõe-se um Elemento Finito Híbrido para simulação do efeito do grau de rigidez rotacional das ligações viga-coluna e 
também se considera o comportamento não linear geométrico de pórticos de aço planos.

Para simulação do comportamento rotacional das ligações viga-coluna, considera-se um modelo linear elástico simplificado, de acordo com as curvas momento-rotação das ligações viga-coluna usuais. A rigidez inicial da ligação, $S_{i n i, i}$, é definida pela inclinação da curva mostrada na Figura 2. De acordo com Faella et al. (2000) a flexibilidade das ligações é medida pela rigidez rotacional inicial, $S_{\text {ini, } i}$.

São apresentados a seguir, os conceitos necessários para definição do Elemento Finito Híbrido, inicialmente para o problema de análise linear elástica e em seguida para análise não linear geométrica.

\subsection{Elemento Finito Híbrido - formulação para análise linear}

Para análise linear elástica a matriz de rigidez do elemento tipo viga plana com extremidades rígidas $\left(K_{i}\right)$ é:

$$
K_{i}=\left[\begin{array}{cccccc}
\frac{E A}{L} & 0 & 0 & -\frac{E A}{L} & 0 & 0 \\
0 & \frac{12 E I}{L^{3}} & \frac{6 E I}{L^{2}} & 0 & -\frac{12 E I}{L^{3}} & \frac{6 E I}{L^{2}} \\
0 & \frac{6 E I}{L^{2}} & \frac{4 E I}{L} & 0 & -\frac{6 E I}{L^{2}} & \frac{2 E I}{L} \\
-\frac{E A}{L} & 0 & 0 & \frac{E A}{L} & 0 & 0 \\
0 & -\frac{12 E I}{L^{3}} & -\frac{6 E I}{L^{2}} & 0 & \frac{12 E I}{L^{3}} & -\frac{6 E I}{L^{2}} \\
0 & \frac{6 E I}{L^{2}} & \frac{2 E I}{L} & 0 & -\frac{6 E I}{L^{2}} & \frac{4 E I}{L}
\end{array}\right]
$$

onde $A$ é a área da seção transversal da barra, $E$ é o módulo de elasticidade e $L$ é o comprimento da barra.

Segundo Simões (1996), a matriz de rigidez do elemento plano que inclui os efeitos das ligações semirrígidas $\left(K_{i}^{S R}\right)$ pode ser obtida a partir da matriz de rigidez do elemento viga clássico, isto é, com extremidades rígidas, modificada por uma matriz de correção $\left(C_{i}\right)$ :

$$
K_{i}^{S R}=K_{i} \cdot C_{i}
$$


A matriz de correção $\left(C_{i}\right)$ considera a alteração da flexibilidade da viga devido a utilização de ligações semirrígidas anexadas nas suas extremidades. Desta forma, alteram-se somente os valores de rigidez flexional, mantendo a rigidez axial inalterada.

De forma prática, utiliza-se o conceito de fator de rigidez $(r)$, como sendo a relação entre a rotação da extremidade da viga devido à aplicação de um momento unitário, e a rotação deste mesmo momento acrescida da rotação devido à flexibilidade da própria ligação (Sánchez e Espín, 2013), Equação (3).

$$
r_{i}=\frac{1}{1+\frac{3 E I}{S_{\text {ini,i }} L}} \quad(i=1,2)
$$

Para ligações teoricamente flexíveis a rigidez rotacional é nula; assim, o valor do fator de rigidez é zero $(r=0)$. Enquanto que, para ligações engastadas, o fator de rigidez é unitário $(r=1)$. Assim sendo, uma ligação semirrígida pode ter um fator de rigidez variando entre zero e um $(0<r<1)$.

Desta maneira, de acordo com Simões (1996), a matriz de correção $\left(C_{i}\right)$, é:

$$
C_{i}=\left[\begin{array}{cccccc}
1 & 0 & 0 & 0 & 0 & 0 \\
0 & \frac{4 r_{2}-2 r_{1}+r_{1} r_{2}}{4-r_{1} r_{2}} & \frac{-2 L r_{1}\left(1-r_{2}\right)}{4-r_{1} r_{2}} & 0 & 0 & 0 \\
0 & \frac{6\left(r_{1}-r_{2}\right)}{L\left(4-r_{1} r_{2}\right)} & \frac{3 r_{1}\left(2-r_{2}\right)}{4-r_{1} r_{2}} & 0 & 0 & 0 \\
0 & 0 & 0 & 1 & 0 & 0 \\
0 & 0 & 0 & 0 & \frac{4 r_{1}-2 r_{2}+r_{1} r_{2}}{4-r_{1} r_{2}} & \frac{2 L r_{2}\left(1-r_{1}\right)}{4-r_{1} r_{2}} \\
0 & 0 & 0 & 0 & \frac{6\left(r_{1}-r_{2}\right)}{L\left(4-r_{1} r_{2}\right)} & \frac{3 r_{2}\left(2-r_{1}\right)}{4-r_{1} r_{2}}
\end{array}\right]
$$

sendo $L$ comprimento do elemento, $r_{1}$ o fator de rigidez da ligação do extremo inicial do elemento e $r_{2}$ o fator de rigidez da ligação do extremo final do elemento.

\subsection{Elemento Finito Híbrido - formulação para análise não linear geométrica}

Nos pórticos de aço são usualmente utilizados perfis estruturais de média ou grande esbeltez, consequentemente, os efeitos advindos do comportamento não linear geométrico da estrutura são significativos e devem ser considerados na análise.

Nesses casos, a variação dos deslocamentos laterais em função da altura da edificação e as ações gravitacionais em virtude do peso próprio e sobrecargas, causam momentos 
secundários que por sua vez provocam deslocamentos adicionais, Figura 3 (a). O Efeito $\mathrm{P}-\Delta$, corresponde ao acréscimo de momentos originados pela deformação estrutural, resultando na mudança do ponto de aplicação das cargas verticais, Figura 3 (b), enquanto que o Efeito $P-\delta$ é referente aos deslocamentos transversais ou flechas em cada elemento da estrutura, (Figura 3 (c)).

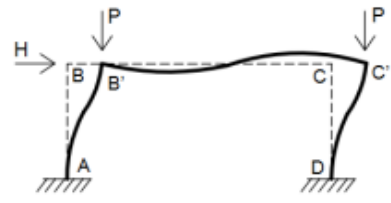

(a) Configuração deformada

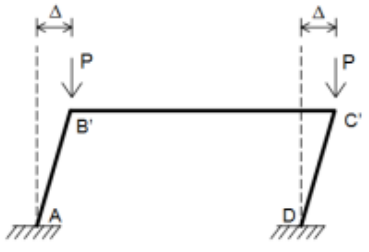

(b) Efeito P- $\Delta$

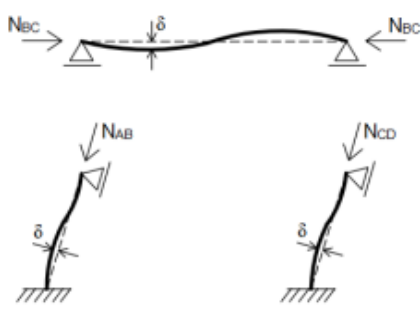

(c) Efeito P-ס

Figura 3 - Efeitos da análise nãolinear

Fonte: Silvestre (2007).

Na sequência, a matriz de rigidez do Elemento Finito Híbrido para a análise não linear geométrica de pórticos de aço $\left(K_{g i}\right)$, foi obtida a partir da matriz de rigidez $\left(K_{i}\right)$, definida na Equação (1). Para tal, consideram-se os efeitos de instabilidade estrutural devido ao comportamento não linear geométrico da estrutura. Desta forma, a matriz de rigidez do elemento $\left(K_{g i}\right)$, é dada por:

$$
K_{g i}=\left[\begin{array}{cccccc}
\frac{E A}{L} & 0 & 0 & -\frac{E A}{L} & 0 & 0 \\
0 & \frac{12 E I}{L^{3}} \phi_{5} & \frac{6 E I}{L^{2}} \phi_{2} & 0 & -\frac{12 E I}{L^{3}} \phi_{5} & \frac{6 E I}{L^{2}} \phi_{2} \\
0 & \frac{6 E I}{L^{2}} \phi_{2} & \frac{4 E I}{L} \phi_{3} & 0 & -\frac{6 E I}{L^{2}} \phi_{2} & \frac{2 E I}{L} \phi_{4} \\
-\frac{E A}{L} & 0 & 0 & \frac{E A}{L} & 0 & 0 \\
0 & -\frac{12 E I}{L^{3}} \phi_{5} & -\frac{6 E I}{L^{2}} \phi_{2} & 0 & \frac{12 E I}{L^{3}} \phi_{5} & -\frac{6 E I}{L^{2}} \phi_{2} \\
0 & \frac{6 E I}{L^{2}} \phi_{2} & \frac{2 E I}{L} \phi_{4} & 0 & -\frac{6 E I}{L^{2}} \phi_{2} & \frac{4 E I}{L} \phi_{3}
\end{array}\right]
$$

Para força normal de compressão $(\mathrm{N}<0)$, tem-se:

$$
\begin{aligned}
& \phi_{1}=\frac{k L}{2} \cot \frac{k L}{2} ; \quad \phi_{2}=\frac{1 k^{2} L^{2}}{12\left(1-\phi_{1}\right)} ; \quad \phi_{3}=\frac{1}{4} \phi_{1}+\frac{3}{4} \phi_{2} \\
& \phi_{4}=-\frac{1}{2} \phi_{1}+\frac{3}{2} \phi_{2} ; \quad \phi_{5}=\phi_{1} \phi_{2}
\end{aligned}
$$

sendo 


$$
k=\frac{\pi}{L} \sqrt{\rho} ; \operatorname{com} \rho=-\frac{N L^{2}}{\pi^{2} E I}
$$

Enquanto que para força normal de tração $(N>0)$, tem-se:

$$
\begin{aligned}
& \phi_{1}=\frac{k L}{2} \operatorname{coth} \frac{k L}{2} ; \quad \phi_{2}=-\frac{1 k^{2} L^{2}}{12\left(1-\phi_{1}\right)} ; \quad \phi_{3}=\frac{1}{4} \phi_{1}+\frac{3}{4} \phi_{2} ; \\
& \phi_{4}=-\frac{1}{2} \phi_{1}+\frac{3}{2} \phi_{2} ; \quad \phi_{5}=\phi_{1} \phi_{2}
\end{aligned}
$$

sendo

$$
k=\frac{\pi}{L} \sqrt{-\rho} ; \operatorname{com} \rho=-\frac{N L^{2}}{\pi^{2} E I}
$$

onde $N$ é força normal que atua no elemento, $L$ o comprimento do elemento, $I$ o momento de inércia e $E$ módulo de elasticidade.

Finalmente, para definição do elemento finito híbrido para o caso não linear geométrico, foram realizadas alterações análogas ao procedimento feito com a matriz de rigidez para o caso de análise linear, $K_{g i}^{S R}=K_{g i} \cdot C_{i}$, utilizando a matriz de correção $\left(C_{i}\right)$, definida na Equação (4).

\subsection{Validação do Elemento Finito Híbrido}

Para a validação do elemento foram confrontados os resultados aqui obtidos com os disponíveis em outras publicações. Foram realizadas simulações computacionais visando quantificar os efeitos do fator de rigidez $\left(r_{i}\right)$, na viga simplesmente apoiada de perfil VE 400X49, com carregamento uniformemente distribuído de $10 \mathrm{kN} / \mathrm{m}$, como ilustrado na Figura 4. Foi adotado módulo de elasticidade do aço igual a 200 GPa. Considerou-se o comportamento não linear e também apoios com ligações flexíveis com fator de rigidez variando de 0 (ligação articulada) a 1 (ligação rígida).

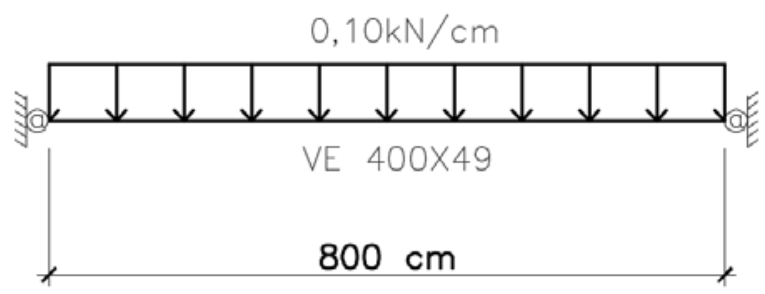

Figura 4 - Viga com ligações semirrígidas. 
Utilizando o programa de análise estrutural desenvolvido, foram obtidos os resultados apresentados na Tabela 1 e comparados com os resultados obtidos por Gomes (2010). Adicionalmente, nas figuras 5 e 6 são apresentadas as variações de rotação e flecha em função do grau de rigidez rotacional das extremidades.

Como pode ser observado na Tabela 1, os valores obtidos no presente trabalho são compatíveis com os estudos de Gomes (2010), onde também foi analisada a variação do fator de rigidez da ligação desde rotulada a rígida.

Tabela 1 - Momentos fletores na viga.

\begin{tabular}{ccccc}
\hline $\begin{array}{c}\text { Fator de } \\
\text { Rigidez } \\
(r)\end{array}$ & \multicolumn{2}{c}{ Gomes (2010) } & \multicolumn{2}{c}{ Presente Trabalho } \\
\cline { 2 - 5 }$\left(M_{\text {ext }}\right.$ & $\begin{array}{c}M_{\text {vão }} \\
(\mathrm{kNcm})\end{array}$ & $\begin{array}{c}M_{\text {ext }} \\
(\mathrm{kNcm})\end{array}$ & $\begin{array}{c}M_{\text {vão }} \\
(\mathrm{kNcm})\end{array}$ \\
\hline Rotulada & 0,0 & 7999,9 & 0,0 & 8000,0 \\
0,1 & 761,2 & 7227,0 & 761,9 & 7238,1 \\
0,2 & 1453,6 & 6538,3 & 1454,5 & 6545,5 \\
0,3 & 2086,2 & 5908,4 & 2087,0 & 5913,0 \\
0,4 & 2666,0 & 5330,4 & 2666,7 & 5333,3 \\
0,5 & 3199,5 & 4798,2 & 3200,0 & 4800,0 \\
0,6 & 3691,9 & 4306,7 & 3692,3 & 4307,7 \\
0,7 & 4147,7 & 3851,4 & 4148,1 & 3851,9 \\
0,8 & 4570,9 & 3428,6 & 4571,4 & 3428,6 \\
0,9 & 4964,9 & 3034,9 & 4965,5 & 3034,5 \\
Rígida & 5332,6 & 2667,4 & 5333,3 & 2666,7 \\
\hline
\end{tabular}

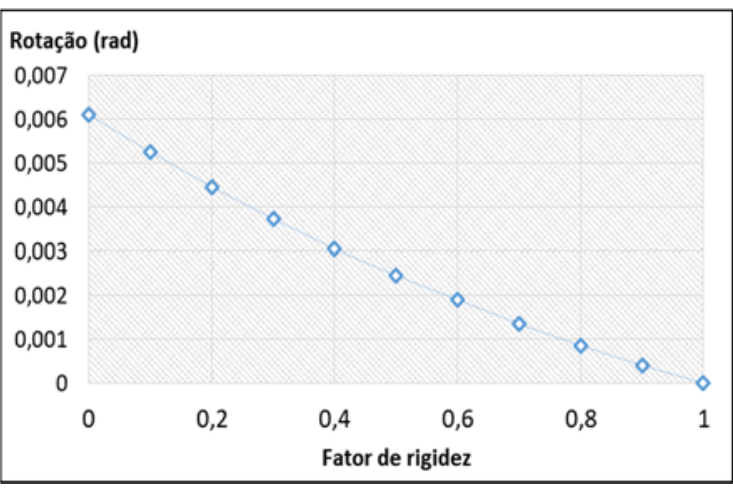

Figura 5 - Rotação nas extremidades da viga.

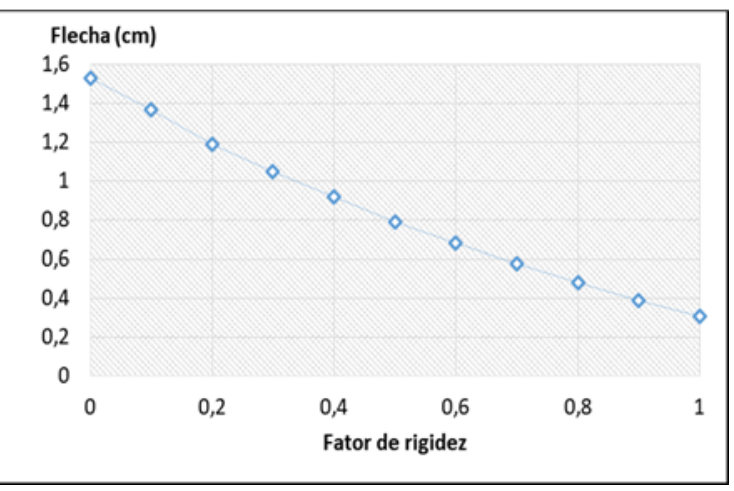

Figura 6 - Flecha no meio do vão da viga.

Observa-se também que o aumento do fator de rigidez da ligação provoca um aumento do momento nas extremidades da viga $\left(M_{\text {ext }}\right)$ e, concomitantemente, uma diminuição do momento no meio do vão $\left(M_{v a ̃ o}\right)$ e que o emprego de ligações semirrígidas permite uma redistribuição de esforços entre os momentos nas extremidades e no meio do vão. 
Examinando a Figura 5, verifica-se que as rotações nas extremidades da viga variam de $q L^{3} / 24 E I=0,00612 \mathrm{rad}$, para ligação articulada $(r=0)$, até um valor nulo, para ligação rígida $(r=1)$.

Analisando a Figura 6, a flecha no meio do vão varia do valor $5 q L^{4} / 384 E I=1,53 \mathrm{~cm}$, para ligação totalmente articulada $(r=0)$, a $q L^{4} / 384 E I=0,306 \mathrm{~cm}$, para ligação idealmente rígida $(r=1)$.

A luz dos resultados mostrados, pode-se afirmar que para uma viga rotulada, a utilização de uma ligação semirrígida possibilita a escolha de um perfil menos robusto, pois o crescimento de momentos nas extremidades da viga diminui a solicitação no meio do vão. E, em comparação a uma ligação rígida, uma ligação menos rígida requer menos detalhes de fabricação e montagem, portanto, mais econômica.

\section{Formulação do problema de otimização}

O modelo matemático para obtenção de pórticos de aço de custo de fabricação mínimo através da determinação de perfis e rigidezes das ligações viga-coluna ótimas, considerando as restrições da norma reguladora nacional, é obtida pela seguinte expressão:

Minimizar

$$
C=C_{\text {perfis }}+C_{\text {ligações }}
$$

Sujeito à

$$
\begin{gathered}
g_{\sigma, i} \leq 1, i=1,2, \ldots, n \\
g_{\delta, j} \leq 1, j=1,2, \ldots, m
\end{gathered}
$$

onde $C$ é o custo total da estrutura, $C_{\text {perfis }}$ é o custo total dos perfis, $C_{\text {ligações }}$ é o custo total das ligações, $g_{\sigma}$ representa as restrições associadas à resistência mecânica do elemento e $g_{\delta}$ representa os deslocamentos nodais, $n$ e $m$ são respectivamente os números de elementos e graus de liberdade restritos da estrutura.

A função objetivo considera o custo de fabricação total da estrutura, a qual deve ser minimizada. O custo total é calculado a partir do somatório do custo dos elementos (vigas e colunas) com o custo das ligações, sendo definido por:

$$
C=c_{s}\left\{\sum_{i=1}^{n c}\left(P_{i} L_{i}\right)+\sum_{i=n c+1}^{n e}\left[P_{i} L_{i}+\sum_{k=1,2}\left(m_{e q, k}\right)\right]\right\}
$$


onde $P_{i}$ é a massa do elemento $(\mathrm{kg} / \mathrm{m}), L_{i}$ o comprimento do elemento, $n c$ o número de colunas da estrutura, $n e$ a quantidade total de elementos da estrutura e $m_{e q, k}$ a massa equivalente da extremidade $\mathrm{k}$ do elemento.

Para essa pesquisa foi escolhida a ligação com chapa de extremidade estendida sem enrijecedores de coluna para todas as ligações viga-coluna. Neste caso, de acordo com Sánchez e Espín (2013), a equação para a massa equivalente é dada por:

$$
m_{e q, k}=43,176+33,5 \times 10^{-5} \cdot R_{k}
$$

\subsection{Restrições de Projeto}

Com a finalidade de projetar estruturas que tenham comportamento semirrígido, foram definidos os limites superior e inferior para rigidez inicial $R_{i}(\mathrm{kNm} / \mathrm{rad})$ das ligações vigacoluna. Em conformidade com a ABNT NBR 8800:2008, o limite inferior (quando a ligação é classificada articulada) e o limite superior (quando a ligação é considerada rígida), são definidas por:

$$
0,5 \cdot E \cdot \frac{I_{v}}{L_{v}} \geq R_{i} \geq 25 \cdot E \cdot \frac{I_{v}}{L_{v}}
$$

onde $E$ é o módulo de elasticidade do aço, $I_{v}$ é o momento de inércia da seção transversal da viga e $L_{v}$ é o comprimento da viga.

Em geral, os elementos que compõem um sistema estrutural estão submetidos a flexocompressão, combinação de esforços que deve ser verificada. A ABNT NBR 8800:2008 define as condições necessárias para que estes sistemas satisfaçam as condições do Estado Limite Último (ELU) e Estado Limite de Serviço (ELS).

Considerando os esforços internos referentes ao problema de flexo-compressão, força axial e momento fletor atuando simultaneamente, esta combinação é definida pelas seguintes inequações:

$$
\begin{array}{ll}
\frac{N_{s d}}{N_{R d}} \geq 0,2 ; & \frac{N_{s d}}{N_{R d}}+\frac{8}{9}\left(\frac{M_{s d}}{M_{R d}}\right) \leq 1 \\
\frac{N_{s d}}{N_{R d}}<0,2 ; & \frac{N_{s d}}{2 N_{R d}}+\frac{M_{s d}}{M_{R d}} \leq 1
\end{array}
$$


onde $N_{s d}$ é a força axial solicitante de cálculo no elemento, $N_{R d}$ é a força axial resistente de cálculo do elemento, $M_{s d}$ é o momento fletor solicitante de cálculo no elemento e $M_{R d}$ é o momento fletor resistente de cálculo no elemento.

De acordo com a ABNT NBR 8800:2008, os deslocamentos máximos admissíveis para as vigas de piso são dados pela seguinte expressão:

$$
\delta_{v}=\frac{L}{350}
$$

onde $\delta_{v}$ é o deslocamento vertical máximo da viga.

Os deslocamentos horizontais máximo $\left(\delta_{h}\right)$ dos nós superiores da coluna em edificações de aço com mais de dois pavimentos, é limitado por:

$$
\delta_{h}=\frac{H}{400}
$$

onde $H$ é a altura da coluna.

\section{Módulo de Otimização}

Na busca de mínimos globais com variáveis de projeto mistas, isto é, com variáveis discretas, continuas e funções de grande não linearidade, foi utilizado o algoritmo genético do Toolbox "Global Optimization" do MATLAB.

Os Algoritmos Genéticos (AGs) são técnicas heurísticas evolucionistas de otimização baseado na evolução das espécies, utilizando operadores inspirados no processo de evolução, Rao (2009). Esses algoritmos são adequados para problemas não lineares, tendo em vista que eles são capazes de avaliar grandes e complexas regiões de busca permitindo a escolha dos melhores indivíduos dentro de uma população de candidatos à solução, Diaz et al. (2011).

O princípio básico dos operadores genéticos é fazer a transformação da população através de sucessivas gerações, imitando o que acontece na natureza. Os operadores genéticos são necessários para a diversificação da população, mantendo as características de adaptação adquiridas nas gerações passadas. 


\section{Exemplos}

Após inúmeras tentativas, os parâmetros da configuração do Algoritmo Genético de otimização para os exemplos aqui apresentados ficaram configurados da seguinte forma: população de 80 indivíduos, máximo de 100 gerações, fração de crossover de 0,85 e taxa de mutação de $10^{-2}$. As especificações do computador LENOVO utilizado para o processamento são: Intel i5 2.4GHz, RAM 4 GB, AMD Radeon Graphics.

\subsection{Exemplo 1}

Esse exemplo foi estudado por Sanchéz \& Espín (2013), e trata-se de um pórtico de dois pavimentos e três vãos. Foram definidos quatro grupos de elementos para a estrutura e mais quatro grupos para as ligações. A geometria da estrutura, os carregamentos e os grupos dos elementos estão representados na Figura 7.

Visando posterior comparação de resultados, para todos os elementos adotou-se o módulo de elasticidade $\mathrm{E}=210 \mathrm{GPa}$, uma tensão de escoamento $f_{y}=250 \mathrm{MPa}$ e o custo unitário do aço de 3,70 reais $/ \mathrm{kg}$.

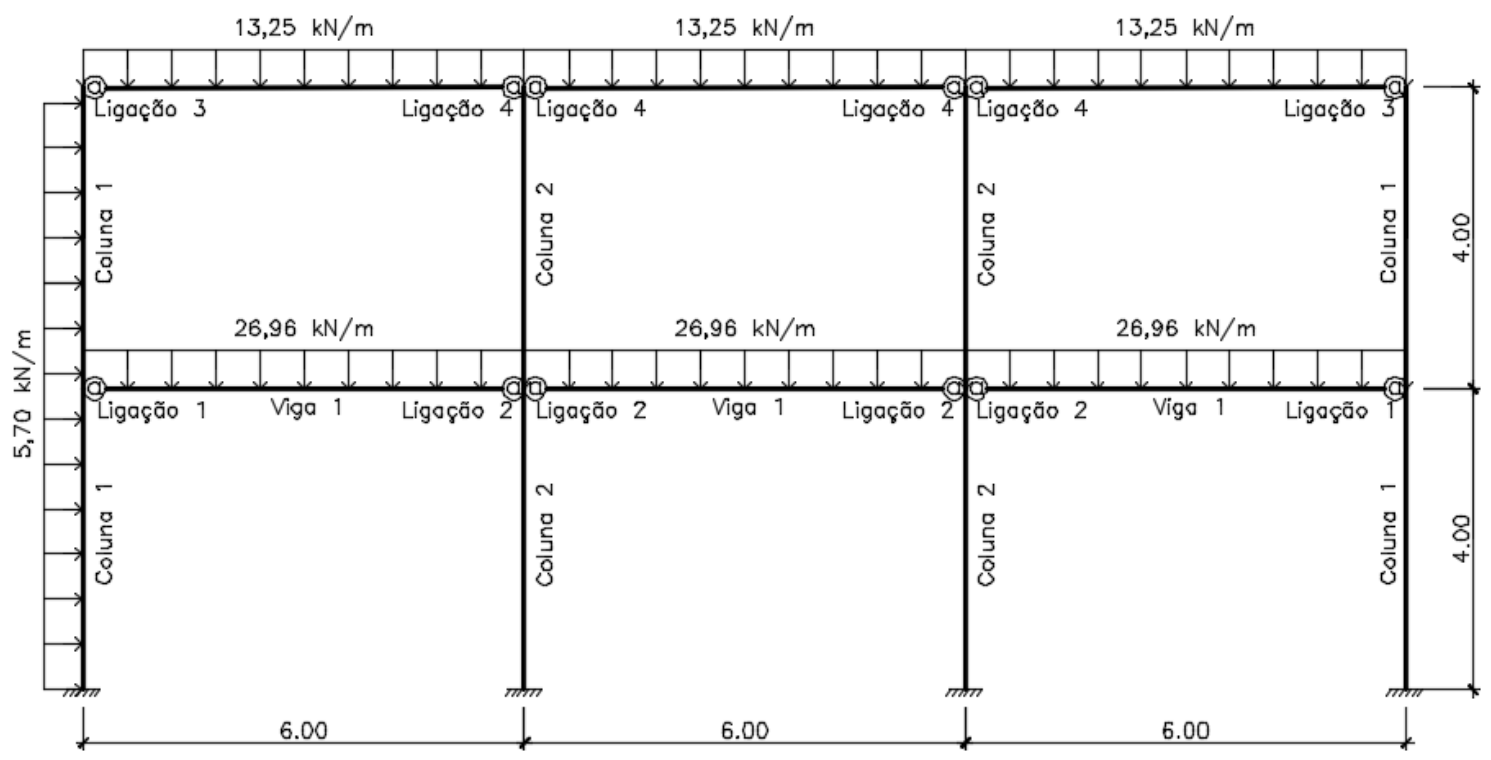

Figura 7 - Pórtico 2: 2 pavimentos e 3 vãos.

Para obtenção da configuração ótima, foram necessárias 48 gerações com um tempo de execução de 65 segundos. Na Figura 8 é apresentado o histórico do processo de otimização com a representação da massa da estrutura a cada iteração até atingir o 
critério de parada. Como resultado ótimo obteve-se uma massa de $2.327,60 \mathrm{~kg}$, que equivale a um custo de $\mathrm{R} \$ 10.476,11$.

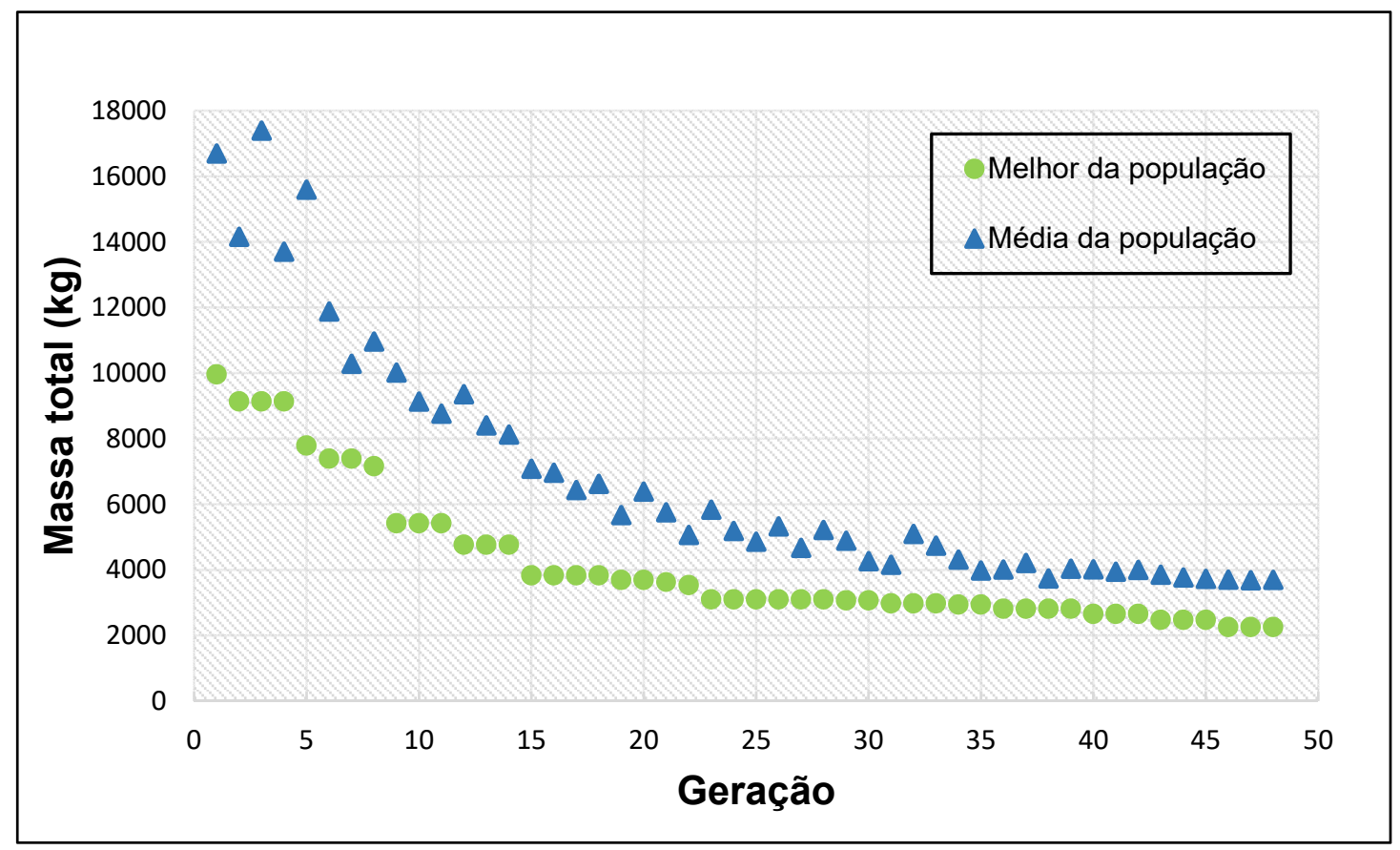

Figura 8 - Histórico do processo de otimização com ligações semirrígidas.

Os perfis estruturais e as rigidezes das ligações ótimas obtidas estão relacionadas na Tabela 2, onde também estão listados os resultados obtidos por Sanchéz e Espín (2013). Nela estão contidas a nomenclatura dos perfis comerciais com sua massa em kg/m em parênteses, como também os valores de rigidez rotacional da ligação e seu respectivo fator de rigidez.

Tabela 2 - Perfis ótimos para cada elemento do pórtico.

\begin{tabular}{ccc}
\hline Variáveis de Projeto & $\begin{array}{c}\text { Sánchez \& Espín } \\
(2013)\end{array}$ & $\begin{array}{c}\text { Presente trabalho } \\
\text { Semirrígida }\end{array}$ \\
\hline Coluna 1 & HEB 120 (26,7) & H $150 \times 22,5$ \\
Coluna 2 & HEB $160(42,6)$ & H $200 \times 35,9$ \\
Viga 1 & IPE $300(42,2)$ & I $310 \times 38,7$ \\
Viga 2 & IPE $240(30,7)$ & I 310 x 38,7 \\
\hline Ligação 1 (kNm/rad) & $14.900(0,63)$ & $10.211(0,53)$ \\
Ligação 2 (kNm/rad) & $20.500(0,70)$ & $12.037(0,58)$ \\
Ligação 3 (kNm/rad) & $7.000(0,63)$ & $30.077(0,77)$ \\
Ligação 4 (kNm/rad) & $16.300(0,80)$ & $16.148(0,64)$ \\
\hline
\end{tabular}


Na Tabela 3 apresenta-se o custo final obtido. Observa-se que foi obtido um custo 4\% menor comparado com os resultados de Sánchez \& Espín (2013), mostrando que a metodologia que aqui se apresenta está condizente com os resultados obtidos na literatura. As rigidezes das ligações também são semelhantes, estando o fator de rigidez dentro do intervalo: 0,53 a 0,80, que corresponde a ligações semirrígidas.

A utilização dos perfis I ao invés dos perfis IPE (europeus) se deu pela criação do banco de dados com perfis comercializados neste país.

Tabela 3 - Custo total do pórtico.

\begin{tabular}{ccc}
\hline Custo (R\$) & $\begin{array}{c}\text { Sánchez \& Espín } \\
(2013)\end{array}$ & Presente trabalho \\
\hline Custo das ligações & $2.153,76(100 \%)$ & $2.154,99(100,1 \%)$ \\
Custo dos perfis & $8.960,29(104 \%)$ & $8.612,12(100 \%)$ \\
Custo total & $11.114,05(103 \%)$ & $10.767,11(100 \%)$ \\
\hline
\end{tabular}

A Figura 9 ilustra graficamente os valores das restrições para a configuração ótima obtida, onde $g(\sigma, 1), g(\delta, 1), g(\sigma, 2)$ e $g(\delta, 2)$ são, respectivamente, esforço interno e deslocamento horizontal máximo das colunas 1 e $2, g(\sigma, 3), g(\delta, 3), g(\sigma, 4)$ e $g(\delta, 4)$ são, respectivamente, esforço interno e a flecha das vigas 1 e 2 e $g(\Delta)$ é a restrição associada ao deslocamento lateral máximo do pórtico. Na solução, as restrições de tensão, flecha e deslocamento lateral ficaram viáveis satisfazendo as normativas locais.

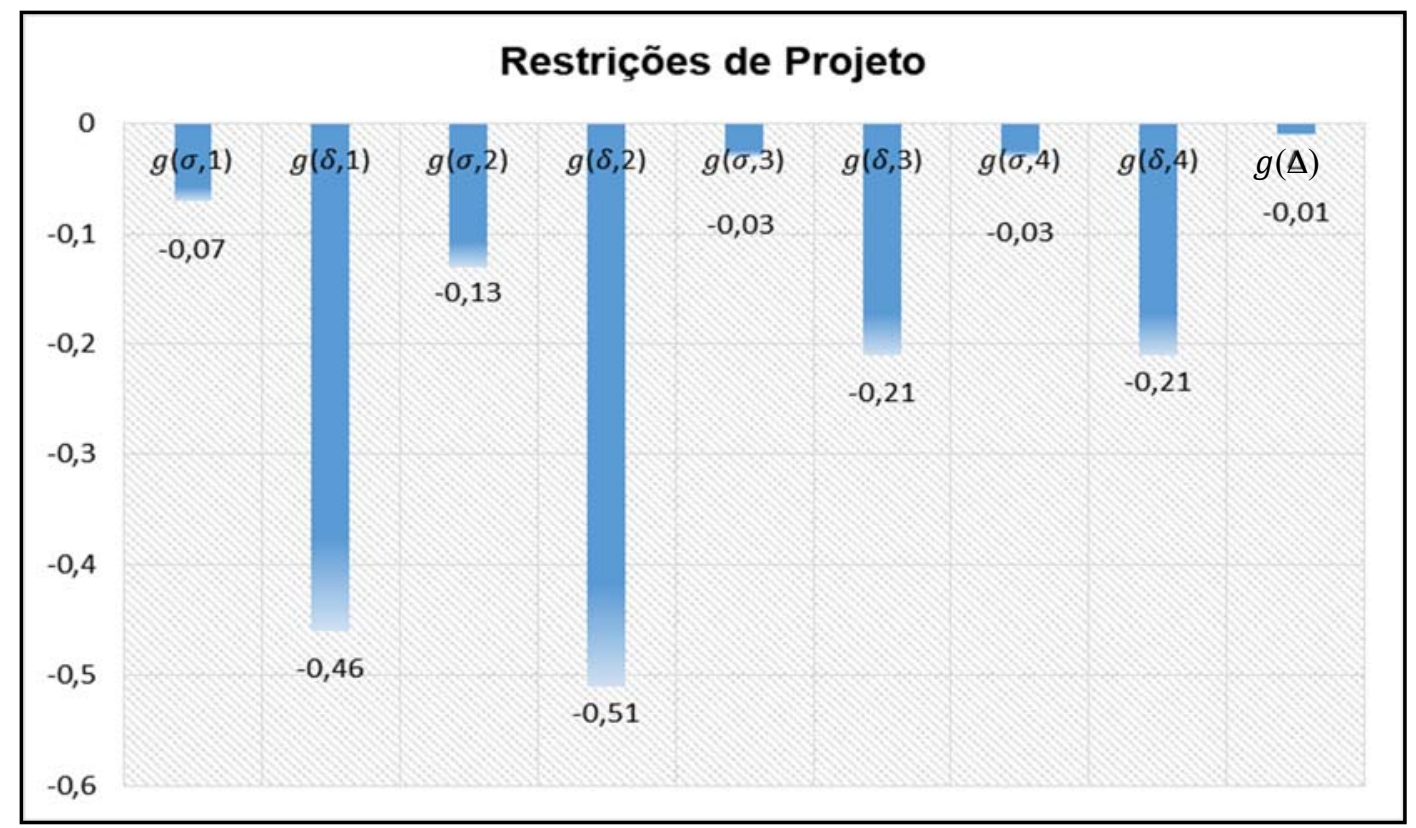

Figura 9 - Restrições de projeto. 
Para esta configuração ótima, as restrições ativas, portanto limitadores do projeto, foram o deslocamento lateral máximo do pórtico $g(\Delta)$ e os esforços internos $(g(\sigma))$ nos elementos 3 e 4, que atingiram aproximadamente $100 \%$ da sua máxima capacidade prescrita em norma, configurando-se como restrições ativas desta aplicação.

\subsection{Exemplo 2}

Esse exemplo foi estudado por Simões (1996), e trata-se de um pórtico plano com 15 elementos, dispostos em três pavimentos e três vão. Foram definidos nove grupos de elementos da estrutura e mais três grupos para as ligações. A geometria, os carregamentos e os grupos dos elementos da estrutura estão representados na Figura 10.

Visando posterior comparação de resultados, para este caso são considerados a tensão de escoamento do aço $f_{y}=250 \mathrm{MPa}$, o módulo de elasticidade $E=206 \mathrm{GPa}$ e o custo unitário do aço de 3,70 reais $/ \mathrm{kg}$.

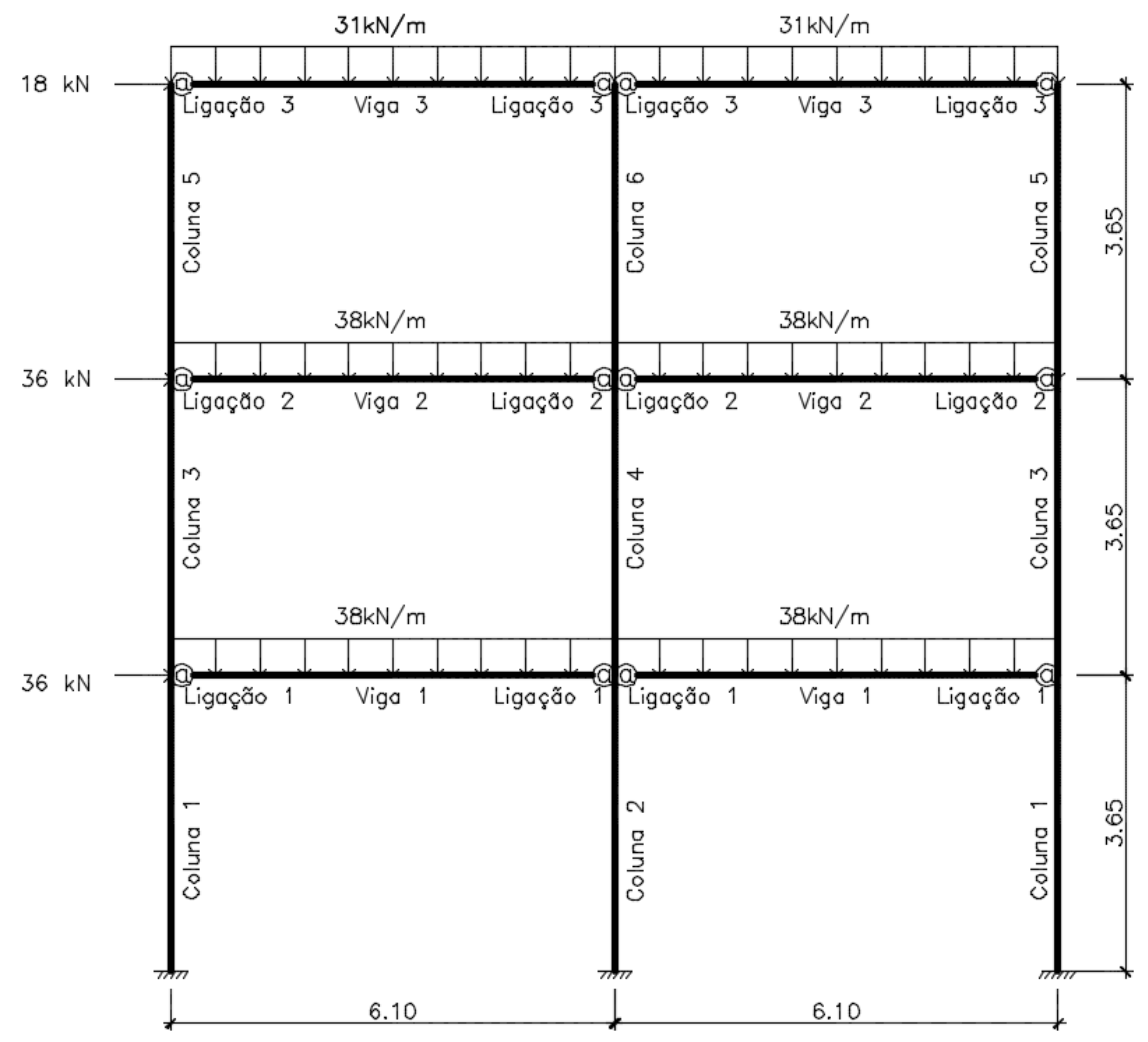

Figura 10 - Pórtico 3: 3 pavimentos e 3 vãos.

Para a convergência dos resultados foram necessárias 46 gerações com um tempo de execução de 62 segundos. Na Figura 11, é apresentado o histórico do processo de 
otimização com a representação da massa da estrutura a cada geração executada até atingir o critério de parada. Como resultado ótimo obteve-se uma massa de 3.998,75 kg, tendo um custo de $\mathrm{R} \$ 14.795,38$.

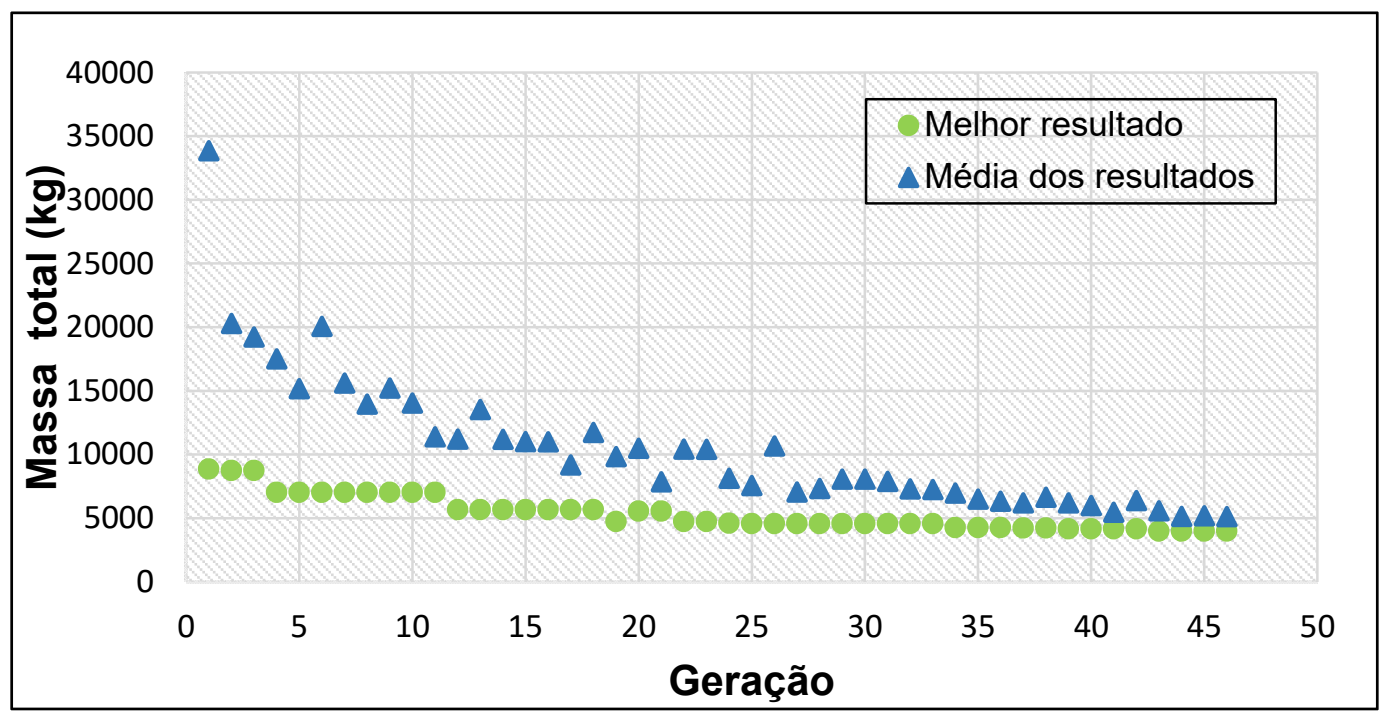

Figura 11 - Histórico do processo de otimização com ligações semirrígidas.

Os perfis da configuração ótima obtida bem como as rigidezes das ligações estão relacionados na Tabela 4, onde também estão listados os resultados obtidos por Simões (1996). Nela estão contidas a nomenclatura dos perfis comerciais com sua massa em $\mathrm{kg} / \mathrm{m}$ em parênteses, como também os valores de rigidez rotacional da ligação e seu respectivo fator de rigidez.

Tabela 4 - Perfis ótimos para cada elemento do pórtico.

\begin{tabular}{ccc}
\hline Variáveis de Projeto & Simões $(1996)$ & $\begin{array}{c}\text { Presente } \\
\text { Trabalho }\end{array}$ \\
\hline Coluna 1 & IPE $360(57,1)$ & I $360 \times 44,6$ \\
Coluna 2 & IPE $550(105,0)$ & I $410 \times 60,0$ \\
Coluna 3 & IPE $330(49,1)$ & H $150 \times 24,0$ \\
Coluna 4 & IPE $330(49,1)$ & I $410 \times 60,0$ \\
Coluna 5 & IPE $300(42,2)$ & I $310 \times 32,7$ \\
Coluna 6 & IPE $240(30,7)$ & I $310 \times 32,7$ \\
Viga 1 & IPE $360(57,1)$ & I $410 \times 60,0$ \\
Viga 2 & IPE $360(57,1)$ & I $360 \times 58,0$ \\
Viga 3 & IPE $330(49,1)$ & | $460 \times 52,0$ \\
\hline Ligação $1(\mathrm{kNm} / \mathrm{rad})$ & $25000(0,60)$ & $12.037(0,54)$ \\
Ligação 2 $(\mathrm{kNm} / \mathrm{rad})$ & $22000(0,575)$ & $30.077(0,58)$ \\
Ligação 3 $(\mathrm{kNm} / \mathrm{rad})$ & $15000(0,55)$ & $16.148(0,61)$ \\
\hline
\end{tabular}


O custo total do pórtico após a otimização está representado na Tabela 5 . O cálculo do custo total da estrutura obtido por Simões (1996) é estimado segundo a Equação 9.

O custo da estrutura ótima obtido foi $9 \%$ menor comparado com os resultados de Simões (1996). Como também pode ser observado na tabela, os valores obtidos para as rigidezes das ligações são bem próximos dos resultados encontrados por Simões (1996).

Tabela 5 - Custo total do pórtico.

\begin{tabular}{ccc}
\hline Custo (R\$) & $\begin{array}{c}\text { Simões (1996) } \\
\text { (Semirrígida) }\end{array}$ & $\begin{array}{c}\text { Presente trabalho } \\
\text { (Semirrígida) }\end{array}$ \\
\hline Custo das ligações & $2.224,40(100 \%)$ & $2.323,23(104 \%)$ \\
Custo dos perfis & $13.875,37(111 \%)$ & $12.472,15(100 \%)$ \\
Custo total & $16.099,77(109 \%)$ & $14.795,38(100 \%)$ \\
\hline
\end{tabular}

A Figura 12 apresenta graficamente os valores das restrições para a configuração ótima obtida, em que $g\left(\sigma_{-} 1\right)$ a $g\left(\sigma_{-} 6\right)$ são os esforços internos das colunas, $g\left(\delta_{-} 1\right)$ a $g\left(\delta_{-} 6\right)$ são os deslocamentos horizontais máximo das colunas, $g\left(\sigma_{-} 7\right)$ a $g\left(\sigma_{-} 9\right)$ são os esforços internos das vigas e $g\left(\delta_{-} 7\right)$ a $g\left(\delta_{-} 9\right)$ são as flechas das vigas e $g(\Delta)$ é o deslocamento lateral máximo do pórtico. Na configuração ótima obtida, todas as restrições de projeto são atendidas $(\mathrm{g}<0)$. No entanto, as restrições ativas $(g<0,1)$ que limitam a redução da função objetivo são os esforços internos das colunas do primeiro pavimento $\left(g\left(\sigma_{-} 1\right)\right.$ e $\left.g\left(\sigma_{-} 2\right)\right)$, a flecha das vigas do terceiro pavimento $\left(g\left(\sigma_{-} 9\right)\right)$ e o deslocamento lateral máximo do pórtico $g(\Delta)$.

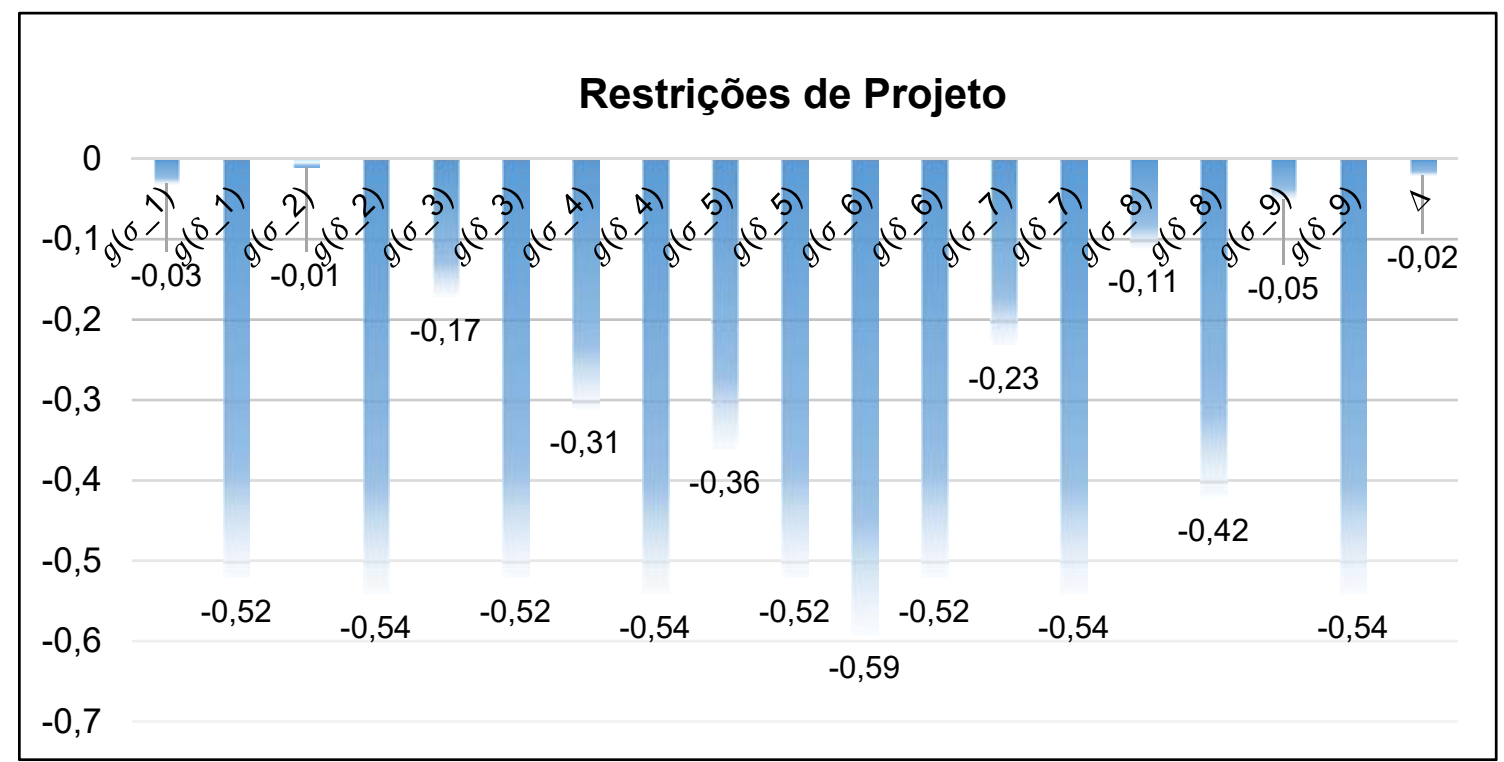

Figura 12 - Restrições de projeto. 


\section{Conclusões}

O estudo mostra a importância do uso de ferramentas computacionais modernas para o dimensionamento ótimo de edificações estruturadas em aço. Foram desenvolvidos dois módulos computacionais para análise e projeto estrutural, integralmente na linguagem computacional MATLAB. Os módulos são independentes, porém integradas entre si de forma harmoniosa através de interfaces computacionais.

A consideração do comportamento semirrígido real das ligações viga-coluna possibilita o aproveitamento mais eficiente dos elementos estruturais, resultando em projetos de menor custo do que quando as ligações são idealizadas como sendo totalmente articuladas ou rígidas, como atualmente ainda é feito.

No módulo de Análise Estrutural foi proposto um Elemento Finito Híbrido a partir do elemento de viga clássico e elementos de mola rotacionais. Verificou-se que o elemento finito proposto representa adequadamente diferentes comportamentos rotacionais das ligações viga-coluna, desta forma atende satisfatoriamente as expectativas da presente pesquisa, apresentando valores coerentes quando comparado com as soluções de outros autores.

Os resultados dos exemplos aqui apresentados indicam que foi possível a redução significativa de custos de fabricação de pórticos planos de aço, principalmente devido a consideração da semirrigidez das ligações viga-coluna notadamente quando comparado com pórticos com ligações rígidas. Assim, foram obtidas configurações ótimas com elementos estruturais mecanicamente mais eficientes.

A metodologia de otimização aqui proposta fornece também uma formulação prática para cálculo do custo dos elementos estruturais e o custo das suas ligações viga-coluna, este último calculado através do conceito de massa equivalente.

Nos resultados obtidos foi observado que a redução de custo de fabricação do pórtico está relacionada tanto à redução dos custos associados aos perfis estruturais mais leves decorrente da consideração do comportamento real das ligações semirrígidas como também da utilização de ligações viga-coluna semirrígidas, que são mais econômicas que as ligações rígidas. 


\section{Agradecimentos}

Os autores agradecem a FAPERJ (Fundação de Amparo à Pesquisa do Estado do Rio de Janeiro).

\section{Referências bibliográficas}

ABNT, NBR 8800:2008. Projeto de estruturas de aço e de estruturas mistas de aço e concreto de edificações, Associação Brasileira de Normas Técnicas, Rio de Janeiro. 2008.

CALFEM: A Finite Element Toolbox, Versão 3.4. Lund University, Lund, v. 4, 2004.

DÍAZ, Concepción; MARTÍ, Pascual; VICTORIA, Mariano. Review on the Modelling of Joint Behavior in Steel Frames. Journal of Constructional Steel Research, Cartagena, v. 67, 2011.

Faella, C; Piluso, V e Rizzano, G. Structural Steel Semi-rigid Connections: Theory, Design and Software. CRC Publisher, Florida, v. 1, p. 36, 2000.

FREITAS, J. P. Dimensionamento ótimo de pórticos de aço considerando ligações semirrígidas e a instabilidade estrutural. Dissertação (Mestrado em Engenharia Civil) - Universidade Estadual do Norte Fluminense Darcy Ribeiro. Campos dos Goytacazes, p.113, 2010.

GOMES, Renata. Análise inelástica avançada de pórticos planos de aço considerando as influências do cisalhamento e de ligações semirrígidas. Tese de Doutorado - Universidade Federal de Minas Gerais. Minas Gerais, p. 143, 2010.

MATLAB. Software Documentation, Versão R2015b. MathWorks, 2015.

MESQUITA, A. C. B. Caracterização e Sistematização do Comportamento Experimental de Ligações Metálicas e Mistas. Dissertação de Mestrado - Faculdade de Ciências e Tecnologia da Universidade de Coimbra, Portugal, 2002.

RAO, Singiresu. Engineering Optimization: Theory and Practice. John Wiley \& Sons, Inc., v. 4, 2009.

SÁNCHEZ, Gregorio; ESPÍN, Antonio Tomás. Design of Planar Semi-Rigid Steel Frames Using Genetic Algorithms and Component Method. Journal of Constructional Steel Research, Cartagena, v. 88, p. 267, 2013.

SILVESTRE, Nuno; CAMOTIM, Dinar. Elastic buckling and second-order behaviour of pitched-roof steel frames. Journal of Constructional Steel Research, Lisboa, v. 63, p. 804, 2007.

SIMÕES, Luis Miguel. Optimization of Frames with Semi-Rigid Connections. Computer \& Structures, Coimbra, v. 60, p. 531-539, 1996. 